\title{
Long-term effects of a single 24-hour maternal separation on three different latent inhibition paradigms
}

\author{
JULIA LEHMANN, CAMILLE LOGEAY, and JORAM FELDON \\ Swiss Federal Institute of Technology, Schwerzenbach, Switzerland
}

\begin{abstract}
Basing animal models for psychiatric diseases on purely environmental manipulations would be a great asset in biological research, such as in the screening of antipsychotic drugs, since they preclude possible interference from pharmacologically induced deficits. Recent investigations have yielded evidence that a single 24-h maternal separation (MS) in rats during the stress hyporesponsive period (SHRP) alters hypothalamic-adrenal-pituitary (HPA) axis response in adult rats and produces long-term behavioral effects, such as deficits in sensorimotor gating (prepulse inhibition) and selective attention (latent inhibition [L]]), which have been related to schizophrenia. Since there are implications that the age of the pups at the time of separation may be a crucial parameter for the neuroendocrine and behavioral effects on adult animals, we investigated the effects of a single 24-h MS on postnatal day (PND) 4, 9, or 18 in unrelated adult male and female Wistar rats on three paradigms of $\mathrm{LI}$ : conditioned taste aversion (CTA), active avoidance (AA), and conditioned emotional response (CER). Whereas there was no effect of MS on CER, MS on PND 9 enhanced LI in the CTA paradigm, and MS on PND 18 disrupted LI in the AA paradigm in males only. Within subjects separated on PND 4, males displayed a severe learning deficit in the AA paradigm, and consequently $L$ was not observed. The results suggest that a single $24-h$ MS, irrespective of the developmental stage of the pups when separated, does not provide a robust animal model for adult disruption of selective attention similar to that observed in schizophrenia.
\end{abstract}

Schizophrenia patients often demonstrate attentional deficits, indicating compromised selective attention (Brebion, Smith, Gorman, \& Amador, 1996; Hemsley \& Zawda, 1976). In the rat, it has been demonstrated that manipulations early in infancy can lead to profound effects on behavior, and therefore it has been suggested that postnatal manipulations may serve as an animal model for human psychiatric diseases, such as attentional disorders. Animal models based purely on behaviorally induced deficits could provide advantages over pharmacological interventions, because they preclude the possibility of drug-drug interactions-for example, when investigating antipsychotic drugs (Geyer, Wilkinson, Humby, \& Robbins, 1993; Varty \& Higgins, 1995). Studies on early environmental manipulations have also been influenced by the human evidence that disturbances in brain development may induce schizophrenia, leading to the neurodevelopmental hypothesis of schizophrenia (Weinberger, 1987). In accordance with this hypothesis, rat models for

The research was supported by a grant from the Swiss Federal Institute of Technology, Zurich. Special thanks are due C. R. Pryce and L. Lacroix for their invaluable help, P. Schmid for technical support, the animal care team for their assistance, and B. Strehler for her help with the preparation of the manuscript. Correspondence concerning this article should be addressed to J. Feldon, Behavioral Neurobiology Laboratory, Swiss Federal Institute of Technology, Schorenstrasse 16, Postfach, CH-8603 Schwerzenbach, Switzerland (e-mail: feldon@) toxi.biol.ethz.ch). schizophrenia and, more specifically, for attentional deficits based on postnatal environmental manipulations have been proposed (Ellenbroek \& Cools, 1998; Feldon \& Weiner, 1992).

Among the paradigms used to investigate attentional deficits and, in particular, deficits of selective attention that have been associated with schizophrenia is that of latent inhibition (LI), a paradigm that can be applied to animals and humans in a very similar manner (Feldon \& Weiner, 1992; J. A. Gray, Feldon, Rawlins, Hemsley, \& Smith, 1991; Lubow \& Gewirtz, 1995). LI is considered to be an index of a subject's ability to ignore irrelevant stimuli and refers to the fact that prior preexposure to a nonreinforced stimulus leads to subsequent retardation of conditioning to that stimulus, as compared with nonpreexposed (NPE) subjects. Schizophrenic patients often demonstrate a disruption of LI (Baruch, Hemsley, \& Gray, 1988; N. S. Gray, Hemsley, \& J. A. Gray, 1992), as do rats after pharmacological manipulations (Ruob, Elsner, Weiner, \& Feldon, 1997; Weiner, Bernasconi, Broersen, \& Feldon, 1997; Weiner, Lubow, \& Feldon, 1984). One of the major purely behavioral manipulations that has been shown to produce changes in selective attention is early handling (EH). This procedure consists of a brief (3$15 \mathrm{~min}$ ) daily separation of the pups from the mother and the siblings from birth until weaning, whereas control subjects are usually left completely undisturbed (nonearly handled, NH). In several studies and across different LI paradigms, it has been demonstrated that $\mathrm{EH}$ enhances LI 
in a sex-specific manner; that is, whereas there is no difference between $\mathrm{EH}$ and $\mathrm{NH}$ females, EH males display enhanced LI, as compared with disrupted LI in NH males (Peters, Gray, \& Joseph, 1991; Weiner, Feldon, \& ZivHarris, 1987; Weiner, Schnabel, Lubow, \& Feldon, 1985). These findings support the more general hypothesis that males may be more susceptible to early perturbations than are females (Erskine, Stern, \& Levine, 1975; Peters et al., 1991; Weinberg, Smotherman, \& Levine, 1978; Weiner et al., 1987). It has also been suggested that $\mathrm{NH}$, which has been considered to constitute a control group, may represent a manipulation in its own right, since it disrupts LI (in males), whereas EH appears to protect against this disruption (Feldon \& Weiner, 1992). However, more recently we have arrived at the conclusion that the enhanced LI observed in EH males is due, at least in part, to the EH manipulation itself, rather than being an effect of NH (unpublished data).

Recently, another form of early environmental manipulation has been introduced-namely, maternal separation (MS), consisting of a prolonged separation of mother and offspring. In a recent study (Lehmann et al., 1998), we reported on the effects of a repeated MS procedure (MS for $6 \mathrm{~h}$ on postnatal days [PNDs] 12, 14, 16, and 18) on selective attention, as measured in three different LI paradigms - namely, conditioned emotional response (CER), two-way active avoidance (AA), and conditioned taste aversion (CTA) - demonstrating that this procedure led to effects similar to those of EH (i.e., it enhanced LI across all three paradigms in MS subjects). Enhancement of LI was due to improved performance in the NPE groups (CTA and two-way AA paradigms) or decreased suppression in the preexposed (PE) group (CER). Contrary to this are the findings reported by Ellenbroek and Cools (1995). They observed disruption of LI measured in the CTA paradigm following a single 24-h MS on PND 10. However, since they employed a single 24-h separation procedure, whereas we used a repeated MS paradigm, it may well be that the opposite results obtained by the two studies are due to the different protocols used. One variable that is potentially important in terms of environmental models is the age/stage of development of the rat pup on exposure to the manipulation (van Oers, de Kloet, \& Levine, 1997). An advantage of MS over EH is that MS provides the opportunity to alter this variable and to expose pups to MS at different stages of development. The effect of the age of pups at separation has been intensively studied in terms of acute endocrine effects, and several recent reports suggest that MS ( $24 \mathrm{~h}$ ) early in the stress hyporesponsive period (SHRP) may lead to effects different from those following MS late in development. Van Oers et al., for example, reported on the effects of MS (24 h) carried out at different pup ages on the hypothalamicpituitary-adrenal (HPA) axis response to a stressor measured on PND 20, finding that MS carried out early in the SHRP (on PND 3) led to a hyperresponsive HPA axis but that MS carried out later in the SHRP (PND 7 or 11) led to a hyporesponsive HPA axis, similar to what has been reported following EH (Levine, 1966; Levine, Haltmeyer, Karas, \& Denenberg, 1967).

Against this background, in the present study we investigated whether the age at separation also plays a critical role in terms of long-term behavioral effects assessed with the LI paradigm. On the basis of the evidence of the disruptive effect of a single 24-h MS on LI reported by Ellenbroek and Cools (1995), we employed a single 24-h MS carried out at three different stages of developmentthat is, at the beginning of the SHRP (PND 4), toward the end of the SHRP (PND 9), and after the SHRP (PND 18). Since Ellenbroek and Cools (1995) reported a disruption of LI in the CTA paradigm following MS on PND 10, we expected to see a similar effect in subjects separated on PND 9. Consequently, in the present study, we first investigated the effects of a single 24-h MS carried out at three different stages of development on LI in the CTA paradigm in adult male and female Wistar rats. Since we were not able to replicate the reported disruptive effect on LI of MS toward the end of the SHRP (PND 9 in our study, PND 10 in that of Ellenbroek \& Cools, 1995), using additional subjects we subsequently employed, with different animals, two further LI paradigms - namely, two-way AA and CER. With regard to sex, we predicted that the effects would be more pronounced in male offspring (information about the sex of subjects is not provided in the Ellenbroek \& Cools, 1995, report), given the evidence for enhanced male susceptibility to behavioral disturbance by the postnatal environment (Erskine et al., 1975; Peters et al., 1991; Weinberg et al., 1978; Weiner et al., 1987).

\section{METHOD}

\section{Subjects}

The experiments were carried out with male and female Wistar rats, all bred in house (Zur:WIST [HanIbm] Animal Services, Swiss Federal Institute of Technology Zurich, Schwerzenbach] and maintained under constant husbandry conditions of reversed cycle lighting (lights on, 19:00-07:00 h) in a temperature- $\left(21^{\circ} \pm 1^{\circ} \mathrm{C}\right)$ and humidity- $(55 \% \pm 5 \%)$ controlled animal facility. The subjects were derived from 64 different litters. Dams were checked twice daily for birth. The day of birth was assigned as PND 0 . All litters were born within a range of 5 days. Within $24 \mathrm{~h}$ after birth, all the litters from which subjects were derived were culled to the same litter size and to a composition of 4 males and 4 females. In order to minimize disturbances to litters that might confound effects of the MS procedure (see below), cage cleaning was carried out once only, at PND 11, for all litters between birth and weaning (PND 21). At weaning, subjects were weighed and placed in group cages (Perspex Macrolon Type IV, $59.0 \times 38.5 \times 20.0 \mathrm{~cm}$ ), 4 same-sex animals per cage, with each group of 4 animals derived from different litters and belonging to the same treatment group. Until testing started, food (Kliba 3430, Klibamühlen, Kaiseraugst, $\mathrm{CH}$ ) and water were available ad lib. For testing, the subjects were divided into three independent groups: 64 animals $(8$ subjects per sex and treatment group) for the CTA paradigm, 64 animals (8 subjects per sex and group) for the two-way AA paradigm, and 64 animals (8 subjects per sex and group) for the CER paradigm. Within group and sex, the subjects were derived from different litters (i.e., they were unrelated). 


\section{Maternal Separation Procedure}

Three experimental groups and one control group were studied. MS subjects were separated from their mothers for $24 \mathrm{~h}, 18: 00$ 18:00 h, at PND 4 (MS4), PND 9 (MS9), or PND 18 (MS18). To carry out MS, the dam was removed to another cage while the pups remained in their home cage. The home cage was then transferred to a separate room and placed on a heat pad set at $33^{\circ} \mathrm{C}$. After $24 \mathrm{~h}$, the pups were returned to the colony room, and the mother was returned to the home cage. Control animals (CON), apart from culling and the single cage cleaning, remained undisturbed with their mothers from birth until weaning.

\section{Experiments}

\section{Conditioned Taste Aversion}

For the CTA procedure, the animals were housed individually in Macrolon cages (Type III, $42.5 \times 26.6 \times 15.0 \mathrm{~cm}$ ) designed in such a way that a drinking tube could be attached and the spout inserted through a hole in the front part of the cage. The water intake of each animal was recorded by measuring the weight of the tube before and after each drinking session.

Animals and Procedure. The experiment used a total of 64 adult 8 -month-old Wistar rats. The animals were transferred from same-sex group caging ( 4 animals per cage) to individual CTA cages. The drinking tubes were removed $48 \mathrm{~h}$ after transferring the subjects to individual cages. During the subsequent 15 days, constituting the entire experiment, the rats were allowed to drink, by reattachment of the drinking tubes, only for $15 \mathrm{~min}$ in the morning (time point between 09:00 and 11:00 h in groups of 16 rats per drinking group) and for $45 \mathrm{~min}$ in the evening (17:00-17:45 $\mathrm{h}$ ). Water consumption of each animal was recorded for every session.

Baseline session. From Days 1 to 5 , the animals were habituated to the schedule of water deprivation.

Preexposure sessions. For preexposure, animals were divided into PE and NPE groups in a counterbalanced manner in relation to their drinking behavior during baseline. From Day 6 to Day 8, all $P E$ animals received a $0.1 \%$ saccharin solution instead of water during the morning session and water in the evening. The NPE animals received water during morning and evening sessions.

Conditioning session. On Day 9 , all the rats received a $0.1 \%$ saccharin solution during the 15-min morning session, followed immediately by an intraperitoneal injection of lithium chloride $(\mathrm{LiCl})$ solution $(0.14 \mathrm{M} ; 1.5 \%$ of body weight).

Rebaseline session. On Day 10, each rat was given a drinking session identical to the baseline sessions (i.e., all the animals received water only).

Test session. The animals were tested over 5 consecutive days, during which all the rats received saccharin during the morning session and water in the afternoon.

Data collection and analysis. Fluid intake was calculated by subtracting the weight of the water tube after a session from its weight before the session. These data were analyzed by a $2 \times 2 \times 4$ $\times 5$ analysis of variance (ANOVA), with main factors of sex (male, female), and preexposure (0,3), MS (CON, MS4, MS9, MS18) and a repeated measurements factor of days $(1-5)$.

\section{Two-Way Active Avoidance}

Apparatus. The apparatus consisted of four identical Coulbourn Instruments (CI) shuttleboxes (Model E10-16TC), each set in a ventilated, sound- and light-attenuating shell (Model E10-20). The internal dimensions of each chamber were $35 \times 17 \times 21.5 \mathrm{~cm}$, as measured from the raised grid floor. The box was divided by an aluminum hurdle ( $17 \mathrm{~cm}$ long, $4 \mathrm{~cm}$ high). The barrier was very thin to prevent animals from balancing on it and thus avoiding shock. Scrambled shocks were delivered from a constant current shock generator (CI, Model E13-14) and scanner (Model E13-13) set at $0.5 \mathrm{~mA}$. The chambers were illuminated during the experimental session with two diffuse light sources (houselights), mounted $19 \mathrm{~cm}$ above the grid floor in the middle of the side walls. The conditioned stimulus (CS) was an $85-\mathrm{dB}[\mathrm{A}]$ tone $(10 \mathrm{sec})$ produced by a $2.9-$ $\mathrm{kHz}$ tone module (Model E12-02) placed behind the shuttlebox on the floor of the shell.

Animals and Procedure. For the two-way active avoidance paradigm, a total of 64 five-month-old rats were tested. The rats were run in squads of four.

The procedure included two stages given $24 \mathrm{~h}$ apart.

Exposure to the apparatus. Each animal was placed in the shuttlebox with the houselight on for a period of $60 \mathrm{~min}$.

Preexposure. Each rat was placed in the experimental chamber. $\mathrm{PE}$ animals received 100 presentations of the CS (10-sec tone), with a variable interstimulus interval of $40 \mathrm{sec}$ ranging from 10 to $70 \mathrm{sec}$. The NPE animals were confined to the chamber for an identical period of time without receiving the tones.

Test. Each animal was placed into the shuttlebox and received 80 avoidance trials on a variable interval schedule of $50 \mathrm{sec}$. Each avoidance trial began with a 10 -sec tone followed by $2 \mathrm{sec}$ of a $0.5-$ $\mathrm{mA}$ shock, the tone remaining on with the shock. If the animal crossed the barrier to the opposite compartment during the tone, the stimulus was terminated, and no shock was delivered (avoidance response). A crossing response during shock terminated the tone and the shock (escape response). If the animal failed to cross during the entire tone-shock trial, the tone and the shock terminated $12 \mathrm{sec}$ after the onset of the tone. The latency to the avoidance or the escape response was recorded in blocks of 10 trials. The measurement started from the presentation of each CS.

Data collection and analysis. The 80 avoidance trials of the test session were divided into eight blocks of 10 trials each. The average latency to avoid or escape from the shock was calculated for each of the eight blocks. These data were analyzed by a $2 \times 2 \times 4$ $\times 8$ ANOVA, with main factors of sex (male, female), preexposure (PE, NPE), and MS (MS4, MS9, MS18, CON) and a repeated measurement factor of blocks (1-8), each consisting of 10 trials.

\section{Conditioned Emotional Response}

Apparatus. The apparatus consisted of four CI test cages (Model E10-10), each set in a ventilated sound-attenuating CI isolation cubicle (Model E10-20). A drinking bottle with a tube opening $3 \mathrm{~mm}$ in diameter was inserted into the chamber through a $3 \times$ $4 \mathrm{~cm}$ hole located in the center of the right wall of the chamber, $1.5 \mathrm{~cm}$ above the grid floor. Licks were detected by a CI infrared optical lickometer (Model E24-01). The experiment was conducted in a dark chamber, and the CS was a 5-sec continuous light (generated from a $28-\mathrm{V}, 40-\mathrm{mA}$ houselight located on the right wall of the chamber, $26 \mathrm{~cm}$ above the grid floor). Shock was delivered through the cage floor from a CI shocker (Model E13-12) and scanner (Model E13-13) set at $0.5 \mathrm{~mA}$.

A CI infrared activity monitor (Model E24-61) was mounted on the ceiling. It was operated in the movement unit mode, in which a 10 -msec pulse is produced each time the monitor detects a change in the animal's infrared heat pattern. This results in a series of pulses (activity counts) at a frequency proportional to the amount of movement made by the animal. Equipment programming and data recording were controlled by a Compaq IBM-compatible personal computer (486/DX2/66).

Animals and Procedure. The experiment was performed on a total of 64 adult 9-month old Wistar rats. One week prior to the start of the experiment, the animals were handled for approximately $3 \mathrm{~min}$ per day, and a $23-\mathrm{h}$ water restriction schedule was initiated. This was followed by 5 days of training, during which each rat was put into the experimental chamber for $20 \mathrm{~min}$. Throughout the experiment, the following data were recorded: total number of licks made by the animal, latency to first lick, and total activity counts. The LI procedure included the following stages.

Preexposure session. Each rat was placed in the experimental chamber and allowed to drink. PE animals received 40 presenta- 
tions of 5-sec steady houselight, with a fixed interstimulus interval of $25 \mathrm{sec}$. The NPE animals were confined to the chamber for an identical period of time without receiving the light stimuli (i.e., in darkness).

Conditioning session. Each rat was placed in the experimental chamber and allowed to drink. Two light-shock pairings were given 5 and $10 \mathrm{~min}$ after the start of the session. Light parameters were identical to those used in preexposure. The 1 -sec shock $(0.5 \mathrm{~mA})$ immediately followed light termination. After the second pairing, the rats were left in the experimental chamber for an additional 5 min.

Rebaseline session. Each rat was given a drinking session identical to the five training sessions.

Test session. The animals were tested on 2 consecutive days. Each animal was placed in the chamber and allowed to drink. The light was presented to each of the 4 rats after it completed 275 licks and lasted for $15 \mathrm{~min}$. The following times were recorded: time to first lick, time to complete licks 1-250, time to complete licks 251 275 (pre-CS), and time to complete licks 276-300 (CS period).

Preexposure, conditioning, rebaseline, and the two test sessions were given $24 \mathrm{~h}$ apart.

Data collection and analysis. The following data were analyzed.

Licking during the rebaseline session: latency to first lick. Latency to first lick during the rebaseline session was submitted to a logarithmic transformation in order to allow a parametric analysis. The data were analyzed by a $2 \times 2 \times 4$ ANOVA, with main factors of sex (male, female), preexposure $(0,20)$, and MS (MS4, MS9, MS18, CON).

Test session: time to complete 25 licks prior to stimulus presentation. The time (in seconds) taken by the animals to make the 25 licks prior to the presentation of the stimulus was analyzed by a $2 \times$ $2 \times 4$ ANOVA with main factors of sex (male, female), preexposure $(0,20)$, and MS (MS4, MS9, MS18, CON).

Test session: time to complete 25 licks after stimulus presentation. The time (in seconds) taken by the animals to make the 25 licks after the presentation of the stimulus was submitted to a logarithmic transformation in order to allow a parametric analysis. The data were analyzed by a $2 \times 2 \times 4$ ANOVA, with main factors of sex (male, female), preexposure $(0,20)$, and MS (MS4, MS9, MS18, CON).

\section{RESULTS}

\section{Conditioned Taste Aversion}

Test session: amount of saccharin consumed. The $2 \times 2 \times 2 \times 5$ ANOVA revealed a significant effect of days $[F(4,192)=79.56, p<.001]$, reflecting an overall decreased taste aversion from Day 1 to Day 5 . The significant main effect of preexposure $[F(1,48)=13.27$, $p<.001$ ] reflects the overall existence of LI-that is, increased saccharin intake in the PE group, as compared with the NPE group. In addition, there was a significant sex $\times$ preexposure $\times$ days interaction $[F(4,192)=4.09$, $p<.01$ ], depicted in Figure 1A, which shows that whereas, within the PE animals, the increased saccharin intake over days was very similar between males and females, in the NPE condition females were faster over days in increasing their saccharin intake (enhanced extinction), as compared with males. In addition, there was a significant effect of preexposure $\times$ MS $\times$ days $[F(12,192)=$ $1.83, p<.05]$, reflecting enhanced LI in MS9 subjects, in comparison with the other three groups. As can be seen in Figure 1B, the enhancement is due mainly to a very strong suppression within the MS9 NPE subjects. The behavior of the four PE groups was very similar. This was. supported by two separate post hoc ANOVAs within the $P E$ and the NPE subjects. No significant effect or interaction involving the treatment or sex factor was obtained within PE subjects (all $p s>.4$ ). Within NPE subjects, the main factor of $\operatorname{sex}[F(1,24)=6.92, p<.02]$ and the sex $\times$ days interaction $[F(4,96)=4.29, p<.005]$ yielded significant results, demonstrating that females extinguish faster than males, and the MS $\times$ days interaction approached significance $[F(12,96)=1.8, p<.06]$, demonstrating that MS9 subjects, irrespective of sex, displayed a strong tendency toward more suppression than did CON, MS4, and MS18 subjects.

\section{Two-Way Active Avoidance}

Latency to avoid or escape. The $2 \times 2 \times 2 \times 10$ ANOVA revealed a highly significant main effect of blocks $[F(7,336)=36.24, p<.001]$, indicating an overall decrease of the latency to avoidance or escape as a function of progressive training. In addition, the analysis revealed a significant main effect of preexposure $[F(1,48)=13.74, p<.001]$, as well as a significant preexposure $\times$ blocks interaction $[F(7,336)=2.75, p<.01]$, supporting the conclusion that, overall, the NPE subjects acquired the avoidance/escape response faster and to a higher level, as compared with the PE subjects - that is, the existence of LI (see Figure 2A). There was a significant effect of $\operatorname{sex}[F(1,48)=4.32, p<.05]$ and a significant sex $\times$ preexposure interaction $[F(1,48)=4.87, p<$ $.04]$, indicating that there was no difference between males and females within the PE subjects but that, within NPE subjects, females displayed decreased latencies (improved avoidance/escape response) when compared with males (PE males, 8.6 \pm 0.2 ; PE females, $8.7 \pm 0.2$; NPE males, $7.9 \pm 0.3$; NPE females, $6.0 \pm 0.2$ ). The MS $\times$ blocks interaction yielded a significant effect $[F(21,336)=1.59$, $p<.05]$, indicating that the four treatment groups (CON, MS4, MS9, and MS18) did not differ in their latencies to respond during the first two blocks of 10 trials but that a difference emerged thereafter; MS9 and MS18 demonstrated shorter latencies to respond (improved avoidance/ escape response), as compared with CON and MS4, which did not differ from each other (see Figure 2B). In addition, there was a significant sex $\times$ preexposure $\times$ MS interaction $[F(3,48)=2.95, p<.05]$, demonstrating normal LI within all female groups but a disruption of LI in MS4 males (owing to a learning deficit in the MS NPE subjects) and MS 18 males (owing to prolonged latencies within PE subjects), which is depicted in Figure 2C.

\section{Conditioned Emotional Response}

Licking during the rebaseline session: latency to first lick. The $2 \times 2 \times 4$ ANOVA yielded no significant main effects or interactions. The overall mean latency to start licking was $54.64 \pm 14.97 \mathrm{sec}$. 
$\mathbf{A}$
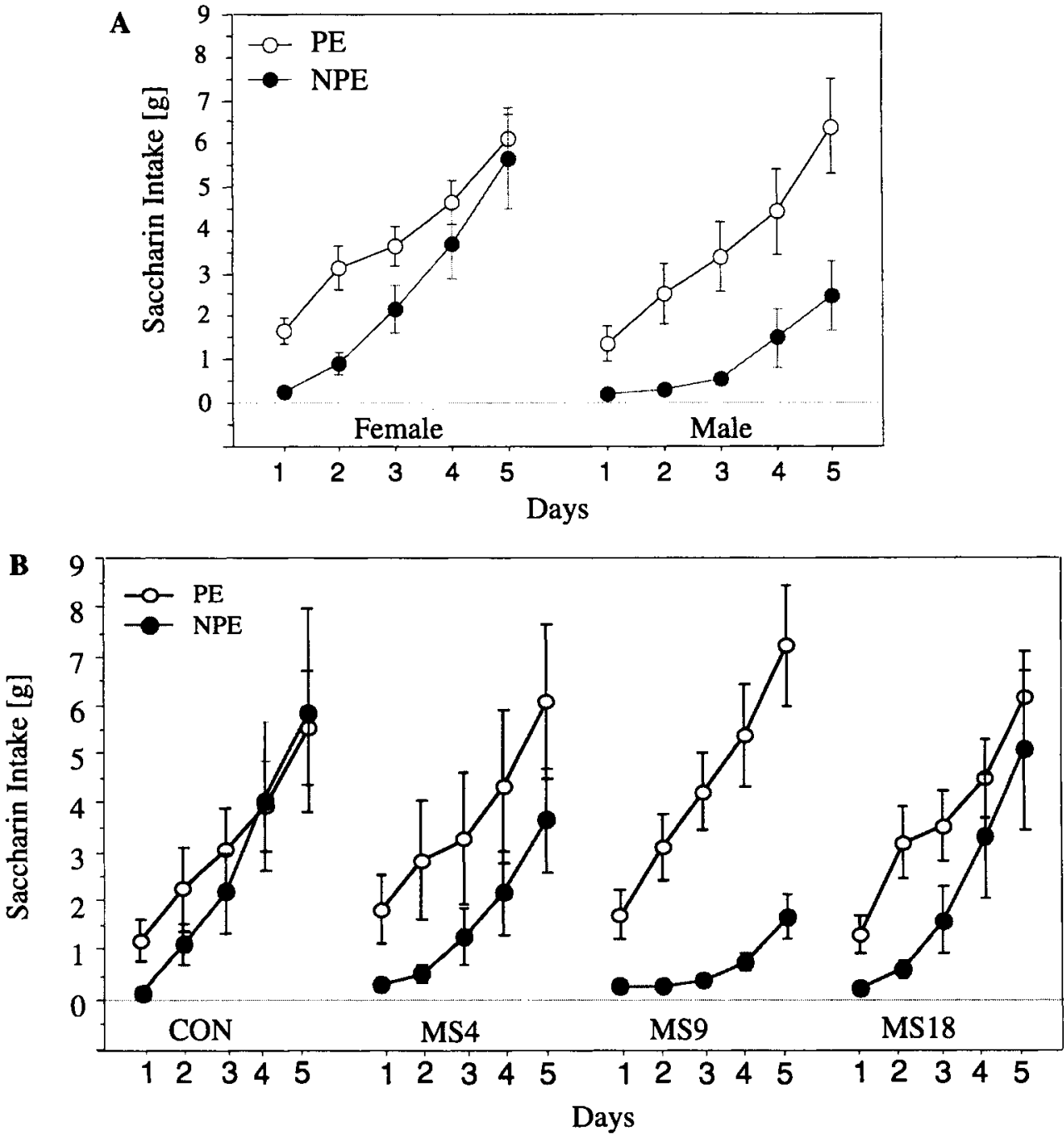

Figure 1. Latent inhibition in the conditioned taste aversion paradigm. (A) Saccharin consumption (in grams) over 5 days of testing for preexposed (PE) and nonpreexposed (NPE) male and female Wistar rats; values are means \pm standard error of the mean; $n$ per group $=16$. (B) Saccharin consumption (in grams) over 5 days of testing for preexposed (PE) and nonpreexposed (NPE) subjects following maternal separation on PND 4 (MS4), 9 (MS9), or 18 (MS18) or following uninterrupted maternal care (control group, CON); values are means \pm standard error of the mean; $n$ per group $=8$.

Licking during the rebaseline session: total licks. A $2 \times 2 \times 4$ ANOVA yielded no significant main effects or interactions.

Test session: time to complete 25 licks prior to the stimulus presentation. The $2 \times 2 \times 4$ ANOVA revealed no significant main effects or interactions. The overall mean time taken to complete licks $251-275$ was $8.48 \pm$ $1.46 \mathrm{sec}$ (mean $\pm S E M$ ).

Test session: time to complete 25 licks after the stimulus presentation. The $2 \times 2 \times 4$ ANOVA revealed a significant effect of preexposure $[F(1,48)=22.77, p<$ $.001]$, demonstrating the overall existence of $\mathrm{Ll}$-that is, a decreased time to complete 25 licks after the onset of the stimulus in the PE group, as compared with the NPE group. The main effect of sex approached significance $[F(1,48)=3.15, p<.09]$, demonstrating that females tended to complete the 25 licks during the stimulus presentation faster than did males. There was no significant main effect or interaction involving the factor of MS (see Figure 3).

\section{DISCUSSION}

The aim of the present study was to assess the effects of a single 24-h MS procedure carried out at different stages of development (early, late, or after the SHRP) on LI, as measured in three different behavioral paradigms in male and female adult Wistar rats. The major findings 
$\mathbf{A}$

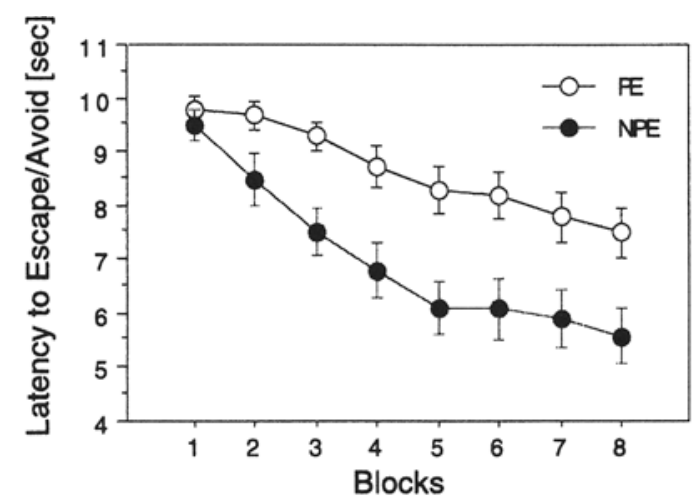

B
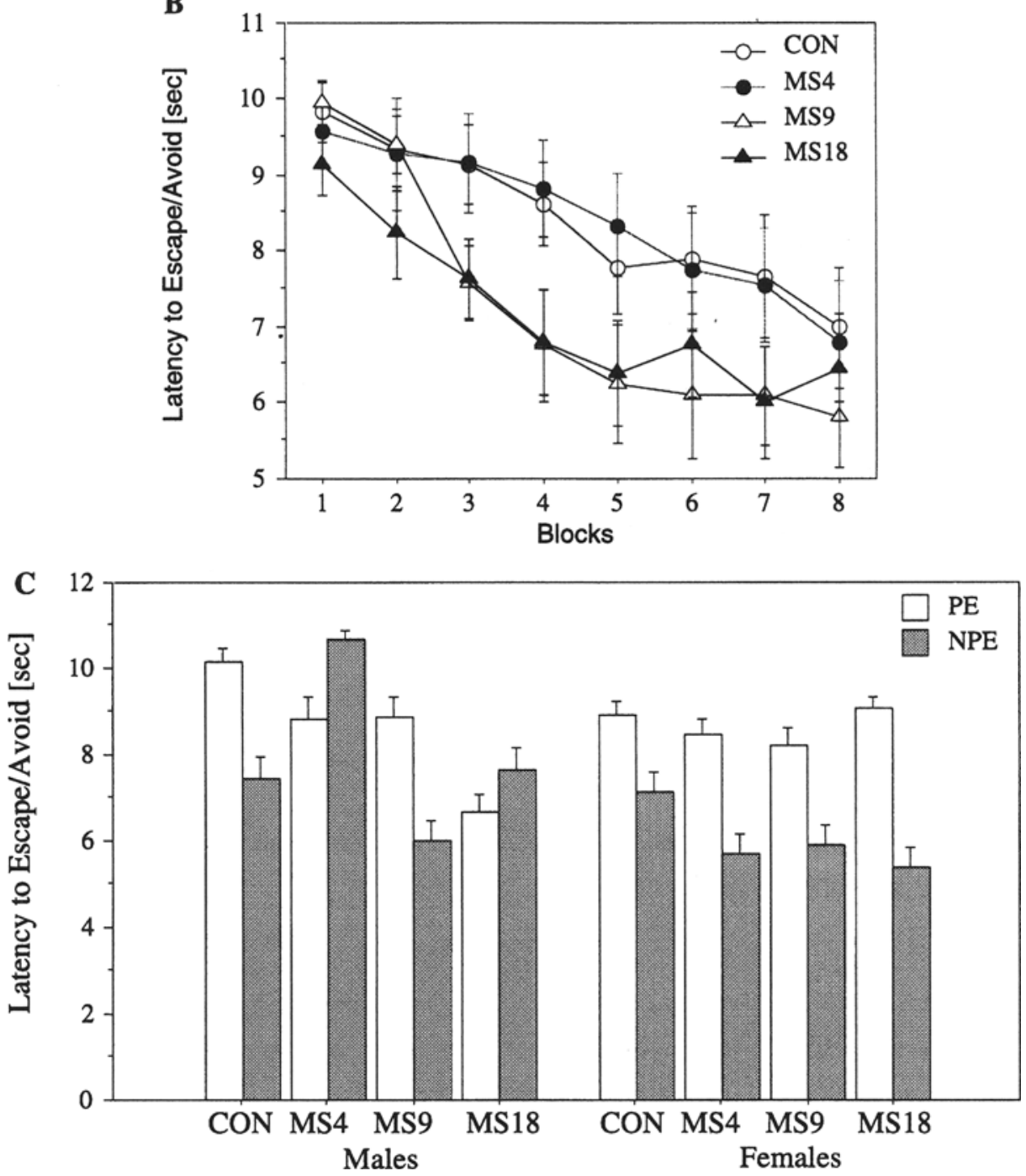

Figure 2. Latent inhibition in the active avoidance paradigm. (A) Latencies to escape/avoid over eight blocks of 10 trials for preexposed (PE) and nonpreexposed (NPE) subjects; values are means \pm standard error of the mean; $\boldsymbol{n}=\mathbf{3 2}$ per group. (B) Latencies to escape/avoid over eight blocks of 10 trials for subjects following maternal separation (MS) on postnatal day 4 (MS4), 9 (MS9), or 18 (MS18) or following uninterrupted maternal care (control group, CON); values are means \pm standard error of the mean; $n=16$ per group. (C) Mean latency to escape/avoid $\pm S E M$ over all blocks collapsed for PE and NPE male and female rats with or without MS; $n=4$ subjects per group. 


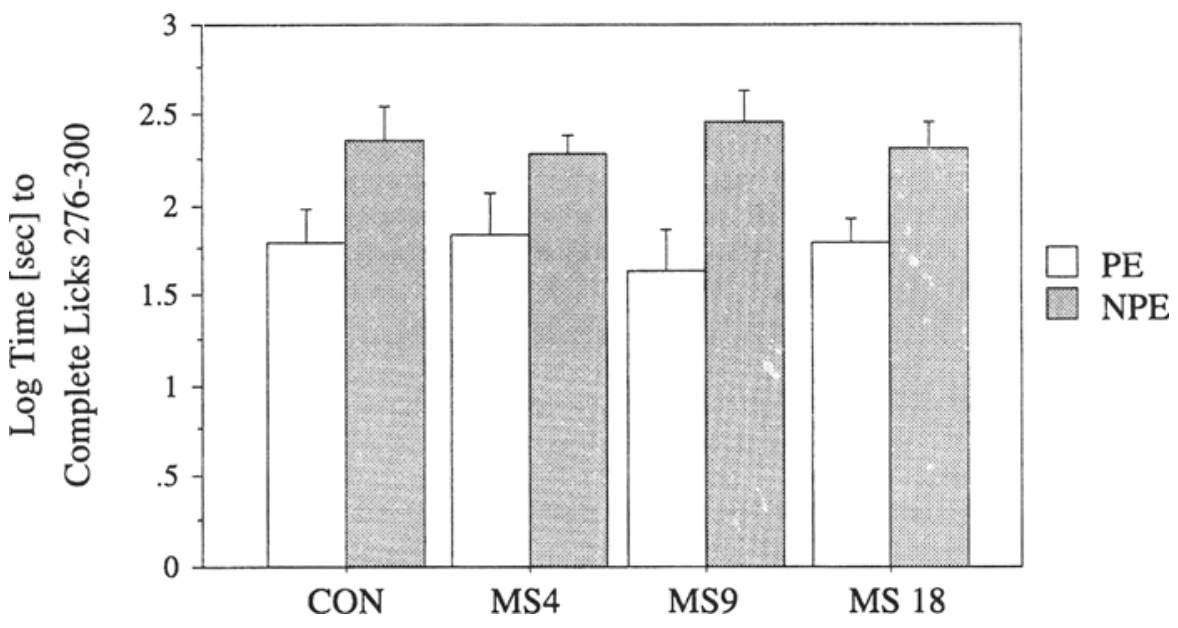

Figure 3. Latent inhibition of the conditioned emotional response paradigm. Time (in seconds) to complete licks $276-300$ (after onset of the conditioned stimulus) logarithmically transformed for preexposed (PE) and nonpreexposed (NPE) subjects following maternal separation (MS) on postnatal day 4 (MS4), 9 (MS9), or 18 (MS18) or following uninterrupted maternal care (control group, $\mathrm{CON}$ ); values are means \pm standard error of the mean; $n=8$ per group.

of this study were as follows. (1) Overall, a robust LI effect was obtained in all three paradigms employed. (2) MS4 did not affect LI in the CTA and CER experiments but led to a disruption of $L I$ in the AA paradigm, mainly because of poor acquisition performance of the NPE males. (3) MS9 did not yield a disruption of LI in any of the three paradigms and, in fact, enhanced LI in the CTA experiment. This was the case in males and females and was due mainly to relatively high suppression in the NPE MS9 subjects. (4) MS18 led to a disruption of LI in the AA paradigm, owing to increased acquisition performance in the PE males, but did not affect LI in CER and CTA. (5) Finally, a robust sex effect was obtained in the AA and CTA paradigms, and there was a tendency toward an effect of sex in the CER paradigm, demonstrating what can be most parsimoniously described as decreased fear in females, relative to males (J. A. Gray, 1987).

The robust $\mathrm{LI}$ effect obtained across all three paradigms demonstrates that the parameters selected within each experiment were totally appropriate for the establishment of LI and the detection of any disruption or facilitation of LI induced by MS. In terms of sex differences, our findings are in line with previous reports in the literature: faster extinction of the CTA effect in females (J. A. Gray \& Lalljee, 1974; Weinberg, Gunnar, Brett, Gonzalez, \& Levine, 1982), lower latencies to respond to the shock in the avoidance-learning paradigm in females (Lehmann, Pryce, \& Feldon, 1999; Saavedra, Abarca, Arancibia, \& Salinas, 1990), and a decreased time to complete 25 licks after stimulus onset in the CER experiment in females (Heinsbroek, Van Haaren, \& Van De Poll, 1988). This high degree of consistency with other reports in the literature, together with the finding of a robust LI effect across all three paradigms, provides a robust validation of the present study and the methodology used.
At the onset of this study, we hypothesized that MS carried out on PND 9 would lead to results similar to those obtained when MS was carried out on PND 10 - that is, a disruption of LI (Ellenbroek \& Cools, 1995). The present study failed to replicate this finding, and indeed, to the extent that MS at PND 9 had an effect on LI, the opposite was the case: MS appeared to enhance LI in the CTA experiment in both males and females, whereas it did not affect $L I$ in the AA and CER experiments. The finding of enhanced LI in the CTA paradigm following MS at PND 9 is largely in line with our previous finding that repeated MS starting on PND 12 (at the end of the SHRP) led to enhanced LI in all three paradigms (Lehmann et al., 1998). Enhanced LI has been also reported following EH, although this was restricted to males (Peters et al., 1991; Weiner et al., 1987; Weiner et al., 1985). However, in the present study LI enhancement was due mainly to high suppression in the NPE subjects, and therefore this cannot be considered to be a real enhancement of LI-that is, one that is due to decreased suppression in PE subjects. Nonetheless, it is clear that MS on PND 9 did not have a disruptive effect on LI in any of the three conditioning paradigms deployed.

Given this discrepancy in findings between the present study and that of Ellenbroek and Cools (1995), it is important to consider some methodological differences between the two. Possible explanations for different outcomes may be age differences, within-strain line differences (Wistar rats were used in both studies), differences in the experimental protocol of the CTA paradigm (Ellenbroek \& Cools's, 1995, CTA experiment consisted of a two-bottle choice paradigm, whereas we used a procedure without the choice component), and differences in the MS procedure per se (e.g., different ambient temperatures during separation of the pups). Zimmerberg and 
Shartrand (1992) have demonstrated that ambient temperature during MS affects development of the dopaminergic system and may, therefore, lead to changes in adult behavior. However, the MS procedure used by these authors was based on a repeated MS for $6 \mathrm{~h}$ per day from PND 2 to PND 15, and direct comparisons must be tentative. The major difference between the Ellenbroek and Cools (1995) study and the one reported here is that our experimental design was based on unrelated subjects, thereby avoiding confounding litter effects (Chapman \& Stern, 1979; Denenberg, 1977; Spear \& File, 1996). Litter effects - that is, treating animals from the same litters as independent data points-decrease variability between subjects within a treatment and therefore increase the likelihood of obtaining significant treatment differences.

Not only for the MS9 condition of our study, but also for MS4 and MS 18 were the effects on LI inconsistent across paradigms. The disruption of LI was obtained only in the AA paradigm and, here, only in MS4 males and MS1 8 males, all females having displayed normal LI. The finding that males, but not females, show an effect of MS is in line with the hypothesis that males are more susceptible to early environmental manipulations than are females, which has been reported previously and for a number of different manipulations (Erskine et al., 1975; Peters et al., 1991; Weinberg et al., 1978; Weiner et al., 1987). However, the disruption of LI in the case of MS4 males was due to a severe deficit of avoidance learning within the NPE subjects (Lehmann et al., 1999), rather than to impaired learning within PE subjects, and therefore does not provide real evidence for a disruptive effect of MS on LI. Such a deficit in avoidance learning in NPE MS males separated on PND 4 is in line with the finding that MS early in the SHRP appears to disrupt aversive conditioning in a conditioned freezing paradigm and has been discussed in more detail elsewhere (Lehmann et al., 1999). A real disruption of $L I$ (i.e., one attributable to the PE subjects) was obtained only in the case of MS18 males, owing to improved performance within the PE subjects. This finding was unexpected, since we hypothesized that a manipulation carried out after the end of the SHRP would not lead to long-term behavioral effects. Although we did not reveal an effect of MS18 in the CTA and CER paradigms, the results obtained in the AA paradigm demonstrate that manipulations starting as late as PND 18 also exert disruptive effects on the development of LI. Other effects of late environmental manipulations on neurodevelopment and behavior also have been reported, including for example, alteration in activity, ethanol selfadministration, and stress reactivity owing to late versus early weaning (Broitman \& Donoso, 1974; Rockman, Hall, Markert, \& Glavin, 1987). Furthermore, we recently reported on the effects of a single 24-h separation on PND 4 and PND 9 in situations of aversive conditioning, providing evidence that to the extent that MS has an effect on adult behavior, MS4 and MS9 show opposite effects (Lehmann et al., 1999).
Taken together, although there was a disruptive effect of MS in the AA paradigm (MS4 and MS 18, males only) and enhancement of LI in the CTA paradigm (MS9), the effects of MS on LI are not consistent across the paradigms, and it is clear from these findings that we must conclude that a single 24-h MS does not represent a robust environmental model of disruption of LI, irrespective of the stage of development at which separation occurred. In terms of long-term behavioral effects, the model of EH versus NH (Feldon \& Weiner, 1992) appears to be more consistent in predicting the effects on selective attention in adulthood.

\section{REFERENCES}

Baruch, I., Hemsley, D. R., \& Gray, J. A. (1988). Differential performance of acute and chronic schizophrenics in a latent inhibition task. Journal of Nervous \& Mental Disease, 176, 578-606.

Brebion, G., Smith, M. J., Gorman, J. M., \& Amador, X. (1996). Reality monitoring failure in schizophrenia: The role of selective attention. Schizophrenia Research, 22, 173-180.

Broitman, S. T., \& Donoso, A. O. (1974). Maternal and sex-related influences on locomotor activity in rats following weaning. Physiology \& Behavior, 12, 309-312.

Chapman, R. H., \& STERN, J. M. (1979). Failure of severe maternal stress or ACTH during pregnancy to affect emotionality of male rat offspring: Implications of litter size effects for prenatal studies. Developmental Psychobiology, 12, 255-267.

DENENBERG, V. H. (1977). Assessing the effects of early experience. In R. D. Myers (Ed.), Methods in psychobiology (Vol. 3, pp. 127-147). New York: Academic Press.

Ellenbroek, B. A., \& Cools, A. R. (1995). Maternal separation reduces latent inhibition in the conditioned taste aversion paradigm. Neuroscience Research Communications, 17, 27-33.

ELLENBROEK, B. A., \& Cools, A. R. (1998). The neurodevelopment hypothesis of schizophrenia: Clinical evidence and animal models. Neuroscience Research Communications, 22, 127-136.

Erskine, M. S., STERN, J. M., \& Levine, S. (1975). Effects of prepubertal handling on shock-induced fighting and ACTH in male and female rats. Physiology \& Behavior, 14, 413-420.

FELdON, J., \& WEINER, I. (1992). From an animal model of an attentional deficit toward new insights into the pathophysiology of schizophrenia. Journal of Psychiatric Research, 26, 345-366.

Geyer, M. A., Wilkinson, L. S., Humby, T., \& RobBins, T. W. (1993), Isolation rearing of rat produces a deficit in prepulse inhibition of acoustic startle similar to that in schizophrenia. Biological Psychiatry, 34, 361-372.

Gray, J. A. (1987). The psychology of fear and stress. Cambridge: Cambridge University Press.

Gray, J. A., Feldon, J., Rawlins, J. N. P., Hemsley, D. R., \& Smith, A. D. (1991). The neuropsychology of schizophrenia. Behavioral \& Brain Sciences, 14, 1-84.

Gray, J. A., \& LALlJEe, B. (1974). Sex differences in emotional behaviour in the rat: Correlation between open field defecation and active avoidance. Animal Behaviour, 22, 856-861.

Gray, N. S., HemsLey, D. R., \& Gray, J. A. (1992). Abolition of latent inhibition in acute, but not chronic, schizophrenics. Neurology, Psychiatry \& Brain Research, 1, 83-89.

Heinsbroek, R. P. W., Van Haaren, F., \& Van De Poll, N. E. (1988), Sex differences in passive avoidance behavior of rats: Sex-dependent susceptibility to shock-induced behavioral depression. Physiology \& Behavior, 43, 201-206.

HeMSLEY, D. R., \& ZAWDA, S. L. (1976). "Filtering" and the cognitive deficit in schizophrenia. British Journal of Psychiatry, 128, 456-461.

LehmanN, J., Pryce, C. R., \& Feldon, J. (1999). The maternal separation paradigm and adult emotionality and cognition in male and female Wistar rats. Pharmacology, Biochemistry \& Behavior, 64, 705-715. 
Lehmann, J., Stöhr, T., Schuller, J., Domeney, A., Heidbreder, C., \& FELDON, J. (1998). Long-term effects of maternal separation on three different latent inhibition paradigms. Pharmacology, Biochemistry \& Behavior, 59, 873-882.

LEVINE, S. (1966). Infantile stimulation and adaptation to stress. In Association for Research in Nervous and Mental Disease (Ed.), Endocrines and the central nervous system (Research Publications, Vol. 43, pp. 280-291). Baltimore: Williams \& Wilkins.

Levine, S., Haltmeyer, G. C., Karas, G. G., \& Denenberg, V. H. (1967). Physiological and behavioral effects of infantile stimulation. Physiology \& Behavior, 2, 55-59.

LuBow, R. E., \& GewIRTZ, J. C. (1995). Latent inhibition in humans: Data, theory and implications for schizophrenia. Psychological Bulletin, 117, 87-103.

Peters, S. L., Gray, J. A., \& Joseph, M. H. (1991). Pre-weaning nonhandling of rats disrupts latent inhibition in males, and results in persisting sex- and area-dependent increases in dopamine and serotonin turnover. Behavioural Pharmacology, 2, 215-223.

Rockman, G. E., Hall, A., Markert, L., \& Glavin, G. B. (1987) Early weaning effects on voluntary ethanol consumption and stress responsivity in rats. Physiology \& Behavior, 40, 673-676.

RuOB, C., ElSNER, J., WEINER, I., \& FELDON, J. (1997). Amphetamineinduced disruption and haloperidol-induced potentiation of latent inhibition depend on the nature of the stimulus. Behavioural Brain Research, 88, 35-41.

Saavedra, M. A., Abarca, N., Arancibia, P., \& Salinas, V. (1990). Sex differences in aversive and appetitive conditioning in two strains of rats. Physiology \& Behavior, 47, 107-112.

Spear, L. P., \& FiLe, S. E. (1996). Methodological considerations in neurobehavioral teratology. Pharmacology, Biochemistry \& Behavior, 55, 455-457.

van Oers, H. J. J., De Kloet, E. R., \& Levine, S. (1997). Persistent, but paradoxical, effects on HPA regulation of infants maternally deprived at different ages. Stress, 1, 249-261.

VARTY, G. B., \& HigGins, G. A. (1995). Examination of drug-induced and isolation-induced disruptions of prepulse inhibition as models to screen antipsychotic drugs. Psychopharmacology, 122, 15-26.

Weinberg, J., Gunnar, M. R., Brett, L. P., Gonzalez, C. A., \& LEviNE, S. (1982). Sex differences in biobehavioral responses to conflict in a taste aversion paradigm. Physiology \& Behavior, 29, 201-210.

Weinberg, J., Smotherman, W. P., \& Levine, S. (1978). Early handling effects on neophobia and conditioned taste aversion. Physiology \& Behavior, 20, 589-596.

WEINBERGER, D. R. (1987). Implications of normal brain development for the pathogenesis of schizophrenia. Archives of General Psychiatry, 44, 660-669.

Weiner, I., Bernasconi, E., Broersen, L. M., \& Feldon, J. (1997) Amphetamine-induced disruption of latent inhibition depends on the nature of the stimulus. Behavioural Pharmacology, 8, 442-457.

Weiner, I., Feldon, J., \& Ziv-HarRis, D. (1987). Early handling and latent inhibition in the conditioned suppression paradigm. Developmental Psychobiology, 20, 233-240.

Weiner, I., Lubow, R. E., \& Feldon, J. (1984). Abolition of the expression but not the acquisition of latent inhibition by chronic amphetamine treatment in rats. Psychopharmacology, 83, 194-199.

Weiner, I., Schnabel, I., Lubow, R. E., \& Feldon, J. (1985). The effect of early handling on latent inhibition in male and female rats. Developmental Psychobiology, 18, 291-297.

Zimmerberg, B., \& SharTrand, A. M. (1992). Temperature dependent effects of maternal separation on growth, activity, and amphetamine sensitivity in rats. Developmental Psychobiology, 25, 213-226.

(Manuscript received May 17, 1999; revision accepted for publication January 19,2000 .) 